\title{
Reflections of health leaders' experiences related dengue solutions from transmitting a technology of larval indices surveillance system in Southern Thailand
}

\author{
Charuai Suwanbamrung* \\ School of Public Health, Walailak University, Nakhon Si Thammarat 80160, Thailand
}

\begin{abstract}
Background: In Thailand, dengue has been spreading for more than 60 years, with a pattern of outbreaks one year followed by two years of normalcy in Surat Thani, Southern Thailand. The "Chaiya model" is the technology of the larval indices surveillance system for dengue solutions that is transmitted to health leaders in the province. Few phenomenology studies of reflection are related to dengue experiences. Therefore, the present study was aimed to explore the reflection of health leaders' experiences related to dengue solutions after the transmitting technology meeting.
\end{abstract}

Methods: Qualitative studies using a phenomenological approach claim that individuals with close proximity to the environment will experience certain phenomena Participants were focused on health leaders participating in the transmitting meeting in 18 districts, as well as three other districts. Two open-ended questionnaires were used for health leaders' reflection. The data analyses used applied a thematic analysis technique including five steps. The trustworthiness technique was used to validate the participants from each area.

Results: Eleven themes that emerged from the reflections of health leaders' experiences included the following: 1) three themes of 18 districts, 2) two themes of the Kanchanadit district, 3) two themes of the Wiang Sa district, and 4) four themes of the Phrasaeng district.

Conclusion: Understanding the overall reflections of health leaders' experiences will have important implications for dengue solutions and specify that individuals participate in all activities. More research should explore this phenomenon in other dengue solution groups in the community such as students and dengue patients.

\section{Introduction}

Dengue is a significant health problem of several countries in tropical and sub-tropical areas. An estimated 50 million dengue infections occur worldwide, and approximately 2.5 billion people live in dengue-endemic countries. Among them, 1.8 billion live in the South-East Asia Region and Western Pacific Region, and 500,000 cases of dengue require hospitalization each year, leading to a death rate of 2.5\% [1-3]. The main risk factors are non-specific drug treatment, adult mosquitoes becoming resistant to chemicals, the mosquito's life cycle becoming shortened due to hot weather, life styles, the density of people per area, and human behavior being barriers to dengue prevention [4-6] The burden from dengue was demonstrated in a study of eight countries in 2005-2006 (including Thailand), revealing that the overall estimated cost per dengue case combining the ambulatory and hospitalized patients, as well as factoring the risk of death, was 828 US\$. The average annual burden for dengue over a five-year period is 465.3 DALYs per million (Disability Adjusted Life Year: DALY) [1]. The World Health Organization (WHO) suggested the integrated vector management (IVM) technique comprising five components: 1) advocacy, social mobilization, and legislation, 2) collaboration within the health sector and with other sectors, 3) an integrated approach to disease control, 4) evidence-based decision making, and 5) capacity-building based on local level and situation analyses [2,7]. Thus, dengue prevention and control need to elucidate the web of causation for dengue, including humans, the environment, mosquitoes, and the dengue virus [8]. According to these suggestions, the transmission of new technological dengue solutions needs to mobilize the health leaders in households and at the district level.

In Thailand, dengue has been spreading for more than 60 years, with a pattern of outbreaks one year followed by two years of normalcy. Surat Thani Province is one of 14 provinces in Southern Thailand that shows a higher rate of infection than the Thai Ministry of Public Health standard (less than 50 cases $/ 100,000$ population). The dengue incidence rates in Surat Thani Province in 2012, 2013, 2014, 2015, 2016 and 2017 were $125,154,64,51,51$, and 92 cases/100,000 population, respectively [9]. The trend of dengue outbreaks from 2012 to 2018 showed no style of outbreak. In 2018, a project concerning the transition to the technology was called "the Aedes aegypti larval indices surveillance system for sustainable dengue solution" and described the successful "Chaiya model" in dengue surveillance in the Chaiya district, Surat Thani Province [10].

${ }^{\star}$ Correspondence to: Charuai Suwanbamrung, Associate Professor, Ph.D., Excellent Center of Dengue Research and Academic Service (EC for DRAS), School of Public Health, Walailak University, Nakhon Si Thammarat 80160 Thailand, Tel: 66814793034; Fax: 6675672106; E-mail: scharuai@wu.ac.th; yincharuai@gmail.com

Key words: reflection, health leaders' experience, dengue solution, transmitting, larval indices surveillance system

Received: January 28, 2019; Accepted: February 18, 2019; Published: February 21, 2019 
The "Chaiya model" is a model of the larval indices surveillance system. The communities received the technology implementing dengue solution strategies to decrease the three traditional Aedes aegypti larval indices such as the Breteau index (BI; the number of positive containers per 100 houses inspected), House Index (HI; the percentage of houses infested with larvae and/or pupae), and Container Index (CI; the percentage of water-holding containers infested with larvae and/or pupae). The technology focused on seven steps of the larval indices surveillance system. First, a household survey was conducted every seven days. Second, the VHV surveyed the larval indices in 1015 households to conduct larval index surveys every $25^{\text {th }}$ day using the "violet book" and sent the larval indices data to the group leader. Third, the group leader collected all of the data from the VHVs in the "blue book" every $28^{\text {th }}$ day. Fourth, the head of each village collected all of the data from the group leaders in the "yellow book". Fifth, the primary health care professionals collected and recorded the data from all the villages using the online program http://surat.denguelim.com, analyzed the data and reported the results on the $30^{\text {th }}$ day of every month. Sixth, the larval indices levels of the BI, HI, and CI were reported at the VHV meeting every month. The health workers then proposed the level of larval indices based on information obtained from all VHVs to prevent dengue in high-risk village areas. Finally, information was communicated by the VHVs to all stakeholders in the district $[10,11]$.

To share the technology, knowledge needs to be transferred to others following the concept of knowledge management, which indicates the systematic management of an organization's knowledge assets to create value and meet tactical and strategic requirements; it consists of the initiatives, processes, strategies, and systems that sustain and enhance the storage, assessment, sharing, refinement, and creation of knowledge [12]. Next, the transmitting Chaiya model shared the Aedes aegypti larval indices surveillance system with health leaders in 18 other districts in Surat Thani Province, focusing on three districts that conduct activities to cover these areas.

Most of the studies with dengue prevention and a control survey with all factors related to dengue outbreaks were quantitative. A few qualitative approaches showed the important themes for dengue solutions. One study was the eco-health model of dengue in Bandung, Indonesia, using unstructured interviews and four focus group discussions. The results showed six principles in four major themes, prevention and control, community participation, housing and surroundings, and weather. The model suggested the theme of the knowledge translation principle that the effective exchange of the dengue knowledge program is important for the health workers, community, and decision makers [13]. The study was associated with health providers' perceptions of dengue in high-risk communities at the urban periphery and center in Machala, Ecuador. The results' themes of the twelve focus group discussions showed that dengue control required a considerable investment of time and resources, and misperceptions included confusion with other febrile diseases, lack of knowledge of the transmission mechanism, and mosquito behavior [14]. These confirm that a qualitative study could present aspects of the health providers' understanding of dengue solutions. One study mentioned that the public health official and community leaders in three districts in Nepal were made of several issuers. Because the results from twenty in-depth interviews and two focus group discussions showed weak diagnostic facilities, private hospitals are not incorporated into the case reporting system and there is an inadequate budget and no routine vector control; however, good community mobilization and an emergency response exist [15].
A phenomenological method focuses on understanding and describing the human experience [16]. An investigative approach was used to understand the lived experience and human experience through their reflections because reflection was used as a method or tool that connects knowledge and experiences [17]. One study focused on the phenomenology approach, namely, travelers' experience when they return home, such as travel health behaviors, dengue prevention, and mosquito avoidance practice. The results of the interview using semistructured open-end questionnaires showed culturally embedded, risk perception of health, and the dengue disease's risk perception influenced travel behavior [18].

As mentioned above, few studies have used the phenomenology approach of health leaders/health providers' reflection of dengue's experiences after the transmitting technology meeting. Therefore, the study was aimed to explore the reflection of health leaders' experience related to dengue solutions after the meeting of the transmitting technology of the larval indices surveillance system in high dengue risk provinces.

\section{Research question}

How does the reflection of health leaders' experience relate to dengue solutions after the meeting of the transmitting technology of the larval indices surveillance system?

\section{Objective}

To explore the reflection of health leaders' experience related to dengue solutions after the meeting of the transmitting technology of the larval indices surveillance system.

\section{Methods}

\section{Study design}

This was a qualitative study that used phenomenological research, which claims that individuals with close proximity to the environment will experience certain phenomenon. Phenomenology relies on latent observation to understand phenomenology based on the underlying features. Natural phenomenology focuses on the belief that the method will shed light on personal knowledge and perceptions to uncover the lived experiences. Thus, phenomenology will be different based on an individual's experience [19], and the experience of all stakeholders involved in solving dengue problems may be different, necessitating the study of the experiences of the leaders involved in dengue fever.

\section{Transmitting technology of the larval indices surveillance system}

The method focused on community participatory action research (CPAR) by experts with successful experiences in dengue solutions. The five steps of the transmitting technology are as follows:

Step I: Community preparation. The research team and health leaders of the prototype district prepared the "Chaiya model" for transmission using the following methods: 1) the technology of the larval indices surveillance system; 2) the "Chaiya model" book that describes the steps of dengue solutions; 3) the computer program http://surat.denguelim.com; 4) a Power Point presentation of dengue knowledge; 5) a book and notebook to record the larval indices surveillance system; and 6) dengue risk assessment forms. In this study, the larval indices surveillance system focused on seven steps of the larval indices surveillance system: 1) a household survey every seven days; 2) the VHV surveyed the larval indices in 10-15 households to 
conduct larval indices surveys every $25^{\text {th }}$ day using the "violet book" and sent the larval indices data to the group leader; 3 ) the group leader collected all of the data from the VHVs in the "blue book"; 4) the head of each village collected all of the data from the group leaders in the "yellow book"; 5) the primary health care professionals collected and recorded the data from all villages using the online program http:// surat.denguelim.com, analyzed the data and reported the results on the $30^{\text {th }}$ day of every month; 6) the larval indices levels of the BI, HI, and CI were reported at the VHV meeting every month. The health workers then proposed the level of larval indices based on information obtained from all VHVs to prevent dengue in high-risk village areas; and 7) information was communicated by the VHVs to all stakeholders in the districts [10].

Step II: The transmitting technology covered the health leaders of 18 districts in Surat Thani province. The district's health leaders were representative leaders from each district, public health offices, subdistrict municipalities, and local administrative organizations. The district chief officer was the chairman of the meeting in each district. The content of the transmitting technology consisted of dengue knowledge (transmission infection, signs and symptoms, treatment, and clinical management) and the larval indices surveillance system (mosquito and larval indices life cycle, dengue prevention and control, surveillance technique, and natural method of dengue prevention)

Step III: The full investigation model included community participatory action research in three districts-Kanchanadit district (covering 117 villages), Wiang Sa district (covering 64 villages), and Phrasaeng district (covering 74 villages). The community meeting planned and conducted the surveillance system in three districts following the seven steps to develop competent dengue knowledge and larval indices.

Step IV: Knowledge sharing with other health leaders in 18 districts of Surat Thani Province. A public health official of the provincial government was the chairman of the meeting (Figure 1).

\section{Participants and selection criteria}

Participants were focused on health leaders participating in the transmitting meeting of the larval indices surveillance system technology. The short reflections of the health leaders' experiences

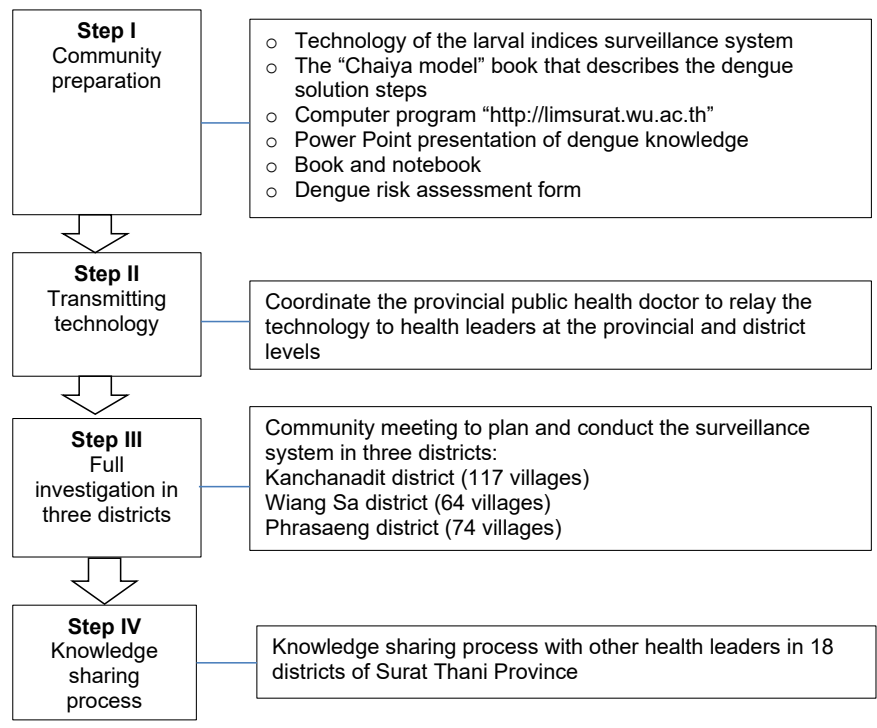

Figure 1. Diagram of the four steps in the transmitting technology strategy were divided into four groups according to the health leaders of the 18 districts, Kanchanadit district, Wiang Sa district, and Phrasaeng district.

\section{Questionnaire guidelines}

The question guidelines were based on the literature review of dengue solutions, larval indices, the surveillance system, and dengue patient treatment. The question guidelines for the open-ended questionnaires were examined by three experts who reviewed them for content validity. The two open-ended questionnaires included questions such as: 1) What is your opinion on sustainable dengue solutions from the transmitting meeting? 2) What are the implementations of the technology in your community? 3) What are the benefits of the transmitting meeting?

\section{Data collection}

The study was approved by "The ethical review committee for research involving human research in Walailak University", ethics code number 59/007. The research team enrolled the participants based on the description of the research's objectives, and the data collection time was 20 minutes per person. The data-collection approach was informal, facilitating the participant's reflection of views and experience. Because the reflections were defined as being associated with relationships and individual needs, they were emotional and personal feelings that have impacted intellectual reflective learning. For example, the study used the reflection technique of personal experience [20].

The data of health leaders' reflections from 18 districts were collected in step II and from all three districts in step III.

\section{Data analysis}

Most of the data analysis in the qualitative study was used thematic analysis [13-15,18]. The thematic structure of a lived experience describes the phenomena in question [21]. Data analysis in this study used the thematic analysis technique including the following five steps: 1) reading and rereading to understand the content from reflections; 2) identifying key words and coding key words; 3 ) setting the category of the key words in relation to their meaning; 4) interpreting the pathology based on meaning and related phenomena; and 5) setting the themes.

\section{Trustworthiness}

At the end of data analysis, the researcher presented the results to the same representative participants so they could review the interpretation thematically. This technique is the most important one to establish a study's credibility [22]. Thus, the participants were asked to validate the thematic and general description of their reflections after the preliminary interpretation.

\section{Results}

The health leaders' reflections in this study showed four sections of health leaders from 18 districts, as well as the Kanchanadit, Wiang Sa, and Phrasaeng districts, in the Surat Thani Province.

\section{Reflections of the health leaders' experience in the 18 districts}

Among the 33 health leaders who provided reflections in the 18 districts, most were women with a mean age of 44 years who remained in the area for more than one year and had a status of village health volunteer or public health official. The three main themes of the health leaders (HLs) were as follows: 
1. "a good model, more benefits, and reduced dengue" indicates that the participants identified the benefits of the knowledge applied to the responsible community such as surveillance, protection against mosquito larvae in all areas, monitoring, and evaluation. They thought the interventions of dengue prevention and controls were better if the dengue knowledge was transmitted to cover all areas in communities. Quotes included the following: "...should be deployed to surveillance, protect mosquito breeding resources in all areas..." ( $\left.\mathrm{HL}_{1}\right)$, "...to apply knowledge to the community that is responsible because it is a very good model..." $\left(\mathrm{HL}_{5}\right) ; "$ "...it is expected that the development of dengue prevention is better than ever..." $\left(\mathrm{HL}_{32}\right)$; and "...this knowledge can be used to control dengue fever in the district as well..." $\left(\mathrm{HL}_{5}\right)$.

2. "satisfied and needed to cover all stakeholders in the community" indicates that the health leaders were satisfied with the meeting regarding aspects such as the knowledge gained, easily understood documents, and the ability to apply the method in the community. However, they needed more dengue knowledge and workshops for village health volunteers and health officials in all subdistricts. The meeting group needed to be small, comprising approximately 10 people per subdistrict, because they could apply the benefits of the program to people in the community. Quotes included the following: "...should organize the local people and relevant agencies for a meeting related to dengue prevention and control, and share the responsibility among households..." ( $\left.\mathrm{HL}_{4}\right)$; "There is a lot of satisfaction in this training, get knowledge, and teachers are friendly with all peoples" $\left(\mathrm{HL}_{11}\right)$; "Village health volunteers can be take the notebook to survey larval indices in the area, and use the program in the health promotion hospital..." $\left(\mathrm{HL}_{12}\right)$.

3. "starting in households, respecting laws, and extending to publicity" indicates that the recipient suggested that all households should participate in the prevention and control of dengue hemorrhagic fever. Importantly, there should be cooperation between government organizations and local health leaders. Moreover, the establishment must occur and be supported with a budget for dengue prevention and control in all sub districts and districts. The village health volunteers must promote that residents take care of their homes, decreasing mosquito breeding both indoors and outdoors. There should be laws governing urban areas, the environment, trash, mosquitoes and mosquito larvae. Quotes included the following: “...need participants from every household and their cooperation for dengue prevention and control" $\left(\mathrm{HL}_{3}\right)$; “...should ask for support from government organizations, local leaders, and establishments" $\left(\mathrm{HL}_{18}\right)$, "should increase the budget for the prevention and control of dengue problem in the area" $\left(\mathrm{HL}_{33}\right)$; “...dengue prevention and control must be done simultaneously in every subdistrict and district" $\left(\mathrm{HL}_{31}\right)$; “...want village health volunteers promoting people for their household management and destruction of mosquito breeding sources in both the home and outdoors..." $\left(\mathrm{HL}_{15}\right)$; and "...must be laws governing urban areas, the environment, trash, mosquitoes and mosquito larvae" $\left(\mathrm{HL}_{8}\right)$.

\section{Reflections of health leaders' experience in the Kanchanadit district}

The Kanchanadit district is a large area including 117 villages and 13 sub-districts. Eighteen key health leaders participated in the meeting and included public health officials, local organization management, municipal employees, community health center employees, and health promotion employees. The Kanchanadit reflection (KR) revealed two themes:

1. "a good method, has a manual, real use, increased continuous meeting and time" indicates that the participants need to be trained and continue with the subgroup, as well as use time from Monday to Friday. Dengue knowledge training was needed in all VHVs with supporting documents and the equipment's surveillance system. Quotes included the following: "need more time to train VHVs" $\left(\mathrm{KR}_{1}\right)$; "There should be additional knowledge in the form of subgroups" $\left(\mathrm{KR}_{10}\right)$; and "The manual can be used to actually meet the objectives because it has larval indices knowledge and shows methods and techniques to immediately solve dengue problems" $\left(\mathrm{KR}_{12}\right)$.

2. "increasing dengue knowledge, disease prevention and model utilities" indicates that the participants have opinions to increase disease prevention by increasing the number of VHVs in the community. Quotes included the following: "should increase VHVs in the village and usually train in dengue prevention" $\left(\mathrm{KR}_{9}\right)$; and "need VHVs to bring knowledge gained in the training to transfer to the community for dengue prevention" $\left(\mathrm{KR}_{19}\right)$.

\section{Reflections of health leaders' experience in the Wiang Sa district}

The Wiang Sa district includes 64 villages in five subdistricts. Fourteen health leaders reflected their experiences. Most of the participants were women with a mean age of 47 years, a mean duration of work in the community of 28 years, and several statuses in the community such as community leader, public health official, nurse, Thai traditional medicine professional, municipal employee, dental public health officer, and village health volunteer. The Wiang Sa reflection (WR) revealed two themes:

1. "need participation from all stakeholders" indicates that the surveillance system for sustainable dengue solutions needs participation from community leaders such as the heads of the villages. VHVs need the participation from leaders and people in the community. Quotes included the following: "the larval indices surveillance system needs participation from all stakeholders" (WR $\mathrm{WR}_{2}$; "head of village must participate because people in the community believe the leader" $\left(\mathrm{WR}_{7}\right)$ and "...community members should add the importance of VHVs" $\left(\mathrm{WR}_{6}\right)$.

2. "continuous meeting to decrease dengue" indicates that the participants needed several dengue solution meetings for VHVs. VHVs should be transferred and suggested to others in the community lacking dengue knowledge such as destroying infested water containers, decreasing the risk of dengue, and predicting dengue outbreaks. Moreover, the community can gain knowledge about budget management for dengue solutions. Quotes include the following: "We would like to arrange intensive dengue solution training for everyone" $\left(\mathrm{WR}_{8}\right)$; and "...the risk of dengue fever is zero...no incident rate of dengue in the community" $\left(\mathrm{WR}_{q}\right)$.

\section{Reflections of health leaders' experience in the Phrasaeng district}

The Phrasaeng district includes 74 villages in eight subdistricts in Surat Thani Province. The 174 participants were VHVs involved in dengue solutions in the district. Most were women with a mean age of 46 years and duration in the area of more than one year. Four themes were revealed from the Phrasaeng reflection (PR):

1. "prevention is better than a solution" indicates that the participant reflected that the dengue problem was overlooked. Dengue solutions need participation from all stakeholders, including households, public health officials, and others in the community. Quotes included the following: "households and village health volunteers must survey 
the larval indices breeding sites every $25^{\text {th }}$, the head of group or zone should collect the data every $28^{\text {th }}$ and send the data to the head of the village on the $30^{\text {th" }}\left(\mathrm{PR}_{70}\right)$; "take knowledge for implementation in the community, and village health volunteers must eliminate larval indices in their houses" $\left(\mathrm{PR}_{169}\right)$.

2. "real knowledge of dengue, and eliminate BI, HI, and CI" indicates that the participants learned about the methods to eliminate mosquitoes, such as the lifestyle of mosquitoes, time of egg incubation to adult mosquito, density of mosquito per square kilometer, and the formula of larval indices (BI, HI, and CI). Quotes included the following: "...increased dengue knowledge and understanding the lifestyle of the mosquito and larval indices such as $\mathrm{BI}, \mathrm{HI}$, and $\mathrm{CI}$ " $\left(\mathrm{PR}_{3}\right)$; "learn to know mosquito breeding sites" $\left(\mathrm{PR}_{100}\right)$; "learn the method Aedes aegypti larval indices including calculating the level of larval indices and the method of implementing elimination..." $\left(\mathrm{PR}_{155}\right)$.

3. "implementation of the surveillance system in the community" indicates that the activities followed the surveillance system because village health volunteers played a role and function based on the household's response. Quotes included the following: "divided village health volunteers into zones such as one village with three zones" $\left(\mathrm{PR}_{19}\right)$; "survey the village health volunteers' households the first time; after that we survey all households and increase knowledge in the community" ( $\left.\mathrm{PR}_{46}\right)$; "back to the community for the referral of dengue knowledge for the larval indices surveillance system, and developing the competency of village health volunteers" $\left(\mathrm{PR}_{31}\right)$.

4. "increasing new methods to use red lime to replace insecticides" indicates that participants mentioned that they were gaining increased dengue information, such as using red lime to replace mosquito insecticide in water and participating in activities for dengue solutions. Quotes included the following: "add new knowledge from the meeting to replace old knowledge and transferring knowledge to people and students" $\left(\mathrm{PR}_{1}\right)$; "discuss with other people in the community and responding to households using red lime" $\left(\mathrm{PR}_{41}\right)$.

\section{Discussion}

Reflections of health leaders' experience from 18 districts revealed the following three themes: "a good model, has benefits, and reduced dengue", "satisfied and needed to cover all stakeholders in the community", and "starting in households, respecting laws, and extending to publicity". Health leaders reflected that the larval indices surveillance system is a good model but needs the participation of all stakeholders. The householders focused on important factors to solve dengue problems. Moreover, community members need to respecting disease prevention laws, as well as local or government policies. The reflections ensured the model will decrease dengue outbreaks and were similar to those in a previous study in the Chaiya district covering 54 villages. The results using the surveillance model showed a decreasing dengue incidence rate $[10,11]$ and were associated with those of a qualitative study of the public health care response to a dengue outbreak in Nepal, concluding that disease surveillance and a response plan need to be area specific [15]. These themes were reflected by representative health leaders making decisions about local policies of dengue prevention at the district level. Moreover, participation is needed in the activities of all steps of the surveillance system, as indicated by the following quote: "...need participants from every household and their cooperation for dengue prevention and control"

Reflections of health leaders' experience in the Kanchanadit district were "a good method, has a manual, real use, increased continuous meeting and time" and "increasing dengue knowledge, disease prevention and model utilities". The reflections of the 18 key health leaders among the public health officials in the district confirmed that the surveillance system and dengue knowledge benefitted dengue solutions. Similarly, the results of a survey study concerning the dengue knowledge of health care professionals showed that they lacked knowledge of the timing of vector bite and diagnosis [23]. They need training knowledge regarding dengue and the larval indices surveillance system; the current status is similar to the confused and misconception of dengue knowledge from the descriptive study of community perceptions of dengue fever and Aedes aegypti [14]. It was confirmed that the technology of the larval indices surveillance system had decreased the dengue problem at the district level $[11,24]$ and in low-risk and high-risk dengue areas in subdistricts [25].

Reflections of health leaders' experience in the Wiang Sa district showed two themes: "need participation from all stakeholders" and "continuous meeting to decrease dengue". The themes reflected the perception of 14 health leaders in the district who focused on the participation of all stakeholders and continuous meeting. In that context, the district set up the larval indices surveillance system and promoted dengue knowledge for VHVs. These themes were associated with the meaning of "participation to overcome professional dominance, to improve strategies and to show a commitment to democratic principles" [26]. Similarly, the theme of the qualitative study with cadres and community leaders was "community participation", which is more multidimensional and very complex in its implementation. Moreover, the conclusion of the study indicates that the dengue solution program required sustainability [13]. Similar to the study of a sustainable dengue solution, experiments were conducted on area more than seven years after setting the larval indices surveillance system [24,27].

Reflections of health leaders' experience in the Phrasaeng district revealed four themes: "prevention is better than a solution", "real knowledge of dengue, and eliminate BI, HI, and CI", "implementation of the surveillance system in the community", and "increasing new methods to use red lime to replace insecticides". The reflections of health leaders in the district were related to the knowledge of dengue and surveillance system and practices in the community. Similarly, a quantitative study showed the relationship of knowledge and practice regarding dengue fever [28], and a qualitative study found that knowledge and attitude subthemes were related to community participation, behavior, and other themes [13]. However, the health leaders reflected on understanding the larval indices types for the success of the transmitting technology of the larval indices surveillance system. If the participants understood the system, the dengue solution could be as sustainable as those in the previous studies $[11,24]$.

\section{Conclusions}

The phenomenology approach of health leaders' reflections was related to the experiences with dengue after the transmitting technology meeting. The results showed the health leaders in the community perceived the technology implementing dengue solution strategies to decrease Aedes aegypti larval indices. Dengue knowledge, the surveillance system, the participation of all stakeholders, and applying the method in many areas are important reflections for dengue solutions.

\section{Limitation and suggestions}

Limitations in this study included the purposive sampling technique regarding participation in the transmitting meeting and the open-end 
questionnaire to record the reflection of each person. Additionally, the study was limited in the generalizability to other areas. The study focused on very specific participants within the meeting and their experiences with dengue solutions. Future research should explore this phenomenon in other dengue solution groups, such as students and dengue patients.

\section{Acknowledgements of support}

The author would like to thank the health leaders who were participants in the study, Walailak University, School of Public Health, and Research and Academic Service (EC for DRAS), School of Public Health, Walailak University, Nakhon Si Thammarat. Moreover, the author extends special thanks to The National Council Research of Thailand, which supported the research project and granted final approval for this study.

\section{Authors' contributions}

The author carried out the study conceptual design, data acquisition, data analyses, and wrote the manuscript in its entirety.

\section{Competing interests}

The author declares that there are no financial or nonfinancial competing interests.

\section{References}

1. WHO (2019) Dengue: Guidelines for diagnosis, treatment, prevention and control. Geneva, Swilzerland: WHO Library Cataloguing-in-Publication data.

2. WHO (2012) Handbook for clinical management of dengue. Genewa, Switzerland: WHO Library Catagologulng-in-Publication Data.

3. Khamim K, Hattasingh W, Nisalak A, Kaewkungwal J, Fernandez S, et al. (2015) Neutralizing dengue antibody in pregnant Thai women and cord blood. PLoS Negl Trop Dis 9: e0003396. [Crossref]

4. Gubler DJ (2011) Prevention and control of aedes aegypti-borne disease: Lesson learned from past successes and failure. Asian Pac J Mol Biol Biotechnol 19: 111-1114.

5. Spiegel J, Bennett S, Gatteraley L, Hayden MH, Kittapong P, et al. (2005) Barriers and Bridges to prevention and control of dengue: The need for a social for a socialecological approach. Eco Health 2: 273-290.

6. Spiegel JM, Bonet M, Ibarra AM, Pagliccia N, Ouellette V, et al. (2007) Social and environmental determinants of Aedes aegypti infestation in Central Havana: Results of a case-control study nested in an intergrated dengue surveillance programe in Cuba. Trop Med Int Health 12: 503-510. [Crossref]

7. WHO (1999) Prevention and control of dengue and dengue hemorrhagic fever: Comprehensive guidelines. New Delhi: WHO Regional Publication, SEARO No. 29.

8. Arunachalam N, Tana S, Espiso F, Kittayapong P, Abeyewickreme W, et al. (2010) Ecobio-social determinants of dengue vector breeding: A multicountry study in urban aand periurban Asia. Bull World Health Organ 88: 173-184. [Crossref]

9. Surat Thani Public Health Office (2018) Situation of epidemiology surveillance report 2012-2017.
10. Suwanbamrung C (2017) Chaiya model: The model of network for Aedes aegypti larval indices surveillance system for sustainable dengue solution from district to province. Nakhon Si Thammarat: Kopon Publication.

11. Suwanbamrung C, Thogian S, Ponprasert C, Situka P, Mopraman P (2018) "Chaiya model": The model of network for Aedes Aegypti larval indices surveillance system for sustainable dengue solution: The results from transmitting technology to community. Area Based Development Research Journal 10: 70-87.

12. Haipjuice (2019) Knowledge Management Tools 2019 [19-01-19]. http://www. knowledge-management-tools.net.

13. Respati T, Feriandi T, Ndoen E, Raksanegara A, Djuhaeni H, et al. (2018) A qualitative ecohealth model of dengue fever (DF) in Bandung, Indonesia. Int J Trop Dis 1: 1-12.

14. Ibarra AMS, Luzadis VA, Cordova MJB, Silva M, Ordonez T, et al. (2014) Asocialecological analysis of community perceptions of degue fever and Aedes aegypti in Machala, Ecuador. BMC Public Health 14: 1135.

15. Griffiths K, Banjira MR, O'Dempsey T, Memslow N, Kroeger A (2013) Public health responses to a dengue outbreak in a Fragile State: A case study of Nepal. J Trop Med 2013: 158462 .

16. Von Eckartsberg R (1988) Introducing existential-phenomenological psychology. New York: Plenum Press.

17. Tashiro J, Shimpuku Y, Naruse K, Maftuhah, Matsutani M (2013) Concept analysis of reflection in nursing professional development. Jpn J Nurs Sci 10: 170-179. [Crossref]

18. Allen KC (2015) Phenomenological analysis of going home in Caribbean-American internation travelers. Trop Dis Travel Med Vaccines 1: 12. [Crossref]

19. Laverty S (2003) Hermerneutic phenomenology and phenomenology: A comparison of historical and methodological considerations. Int J Qual Methods 2: 21-35.

20. Suwanbamrung C (2015) Learning experience of student nurses though reflection on clinical practice: A case study in pediatric nursing, southern Thailand. Walailak Journal Sci \& Tech 12: 623-629.

21. Christofi V, Thompson C (2007) You cannot go home again: A phenomenological investigation of returning to the sojourn country after studying abroad. J Couns Dev 85: 53-63.

22. Flick U, Lardorff Ev, Steinke I (2004) A companion to qualitative research. London: SAGE Publications.

23. Hamid SM, Siddiqui MI, Qutub RAM, Raees F, Hashmi S (2017) Knowledge of dengue fever among health care professional in large hospitals of Makkah al Mukarramah. JBUMDC 7: 209-213.

24. Suwanbamrung C, Thoutong C, Eksirinimit T, Supapon T, Thongkew K (2018) The Use of "Lansaka Model" as the Larval indices surveillance system for a sustainable solution to the dengue problem in southern Thailand. JBUMDC 13: e0201107.

25. Suwanbamrung C (2018) Developing the active larval indices surveillance system for dengue solution in low and high dengue risk primary care units, Souhern Thailand. $J$ Health Res 32: 408-420.

26. Baum F, MacDougall C, Smith D (2006) Participatory action research. $J$ Epidemiol Community Health 60: 854-857.

27. Suwanbamrung C (2018) Social engagament-scholarship in dengue problem solution A case of developing routine work into area based collaborative research "Lan Saka" and "Chaiya" model. Journal of Community Development and Life Quality 7: 45-56.

28. Harapan H, Bustaman A, Radiansyah A, Angraini P, Fasli R, et al. (2017) Dengue prevention: Confirmatory factor analysis of relationships between economic status, knowledge, attitudes and practice, vaccine acceptance and willingness to participate in the study. Southeast Asian J Trop Med Public Health 48: 297-305. [Crossref]

Copyright: (02019 Suwanbamrung C. This is an open-access article distributed under the terms of the Creative Commons Attribution License, which permits unrestricted use, distribution, and reproduction in any medium, provided the original author and source are credited. 